\title{
Oral Mucosal Injury in Oncology Patients
}

\author{
Douglas E. Peterson
}

Published online: 3 August 2014

(C) Springer International Publishing AG 2014

\begin{abstract}
The field of oral mucosal injury caused by cancer therapies has strategically matured in the scientific as well as clinical realms over the past decade. Prior to the late 1990s, the condition had been viewed by many healthcare providers as an inevitable consequence of high-dose chemotherapy and other intensive therapies in oncology practice. Fortunately, expanding insights into the pathobiology as well as design strategies for clinical trials in recent years have fostered development of high-quality clinical practice guidelines as well as health professional education relative to prevention and treatment. This report is directed to analysis of this contemporary modeling. Guideline-based management recommendations that have emerged over the past year and controversies in the field are highlighted.
\end{abstract}

Keywords Oral mucosal injury · Oncology $\cdot$ Pathobiology · Management guidelines

\section{Introduction}

Oral mucositis continues to be a clinically significant toxicity in many patients undergoing high-dose chemotherapy $[1,2$, $3 \bullet \cdot, 4-11]$. The condition can cause severe oral pain with resultant need for systemic narcotics, hospitalization, and total parenteral nutrition. Oral functions including speaking, eating,

\section{E. Peterson $(\square)$}

Section of Oral Medicine, Department of Oral Health \& Diagnostic

Sciences, School of Dental Medicine, University of Connecticut

Health Center, Farmington, CT 06030-1605, USA

e-mail: Peterson@nso.uchc.edu

\section{E. Peterson}

Program in Head \& Neck Cancer and Oral Oncology, Neag Comprehensive Cancer Center, University of Connecticut Health Center, Farmington, CT, USA and swallowing as well as maintenance of oral hygiene can be compromised. Myelosuppressed cancer patients such as those individuals undergoing high-dose chemotherapy induction for leukemia are at additional risk for mucosal infection, sepsis, and death [4]. Despite these adverse consequences, oral mucositis continues to be an important unmet medical need, with molecularly targeted mucositis management approved by the US Food and Drug Administration (FDA) for only a relatively limited number of patients [12].

Over the past 15 years there have been highly impactful advances in delineating the fundamental pathobiology associated with oral mucositis in patients undergoing intensive cancer treatments $[5,10,11,13,14]$. While several of the characteristics share similarity with other models such as inflammatory bowel disease [11], there are unique features as well, such as impaired basal stem cell epithelial injury, that are central to the toxicity. Moreover and as described below, a unique expression of oral mucosal injury has arisen in recent years in association with targeted cancer therapies $[15,16 \bullet \bullet$, 17]. The fundamental mechanisms that contribute to causation and healing remain to be elucidated.

The economic cost of managing oral mucositis and its sequelae has also emerged in recent years as a fundamentally key issue relative to utilization of healthcare resources. Estimates have ranged from approximately US $\$ 18,000$ in head and neck cancer patients [18] to more than US\$42,000 in hematopoietic cell transplant (HCT) patients [19]. In this context, it is vital that state-of-the-science management approaches, based on high-quality basic, translational, and clinical research, be utilized in the clinical setting.

Having said this, there are several barriers to achieving this ideal vision. As delineated in this report, these impediments range from continued need for pathobiologic modeling to assessment of impact of utilization of high-quality, evidencebased guidelines in clinical practice. 


\section{Pathobiology}

The contemporary model of pathobiology, first delineated by Sonis in 1998, comprises a five-phase model of mucosal injury and subsequent healing [20-22]. This conceptual framework at the time represented a creative and impactful departure from the classic modeling of oral mucositis, which until then was characterized by the seemingly exclusive role of basal epithelial stem cell injury caused by cancer therapy.

This landmark model as reported in 1998 has continued to mature as additional research has been produced further insights into the paradigm, with further understanding of the up-regulation of reactive oxygen species in the early hours of exposure to cytotoxic chemotherapy. This ubiquitous tissue response to injurious stimuli is then followed by increased expression over the next 24-96 hours of proinflammatory cytokines such interleukin (IL)-1 $\beta$ and IL-6 and tumor necrosis factor- $\alpha$, as well as injury to the microvasculature $[13,14]$. Damage to the extracellular matrix also contributes to the pathobiology [23]. This matrix is a complex structural network comprising fibrous proteins, proteoglycans, and glycoproteins, and is key in preserving regulation of normal tissue morphology and wound healing.

External factors unique to the oral or gastrointestinal environment, such as the altered colonizing microbial ecosystem, can further affect this trajectory $[23,24]$. The contemporary model of oral mucositis pathobiology thus continues to be viewed as a complex, interdependent interplay among genetic governance of risk, inflammation biology, oral microbiome, and tissue healing involving both epithelium as well as submucosa.

The central role of genetic polymorphisms in this dynamic has become increasingly prominent in recent years as well $[5$, 11]. Unlike hypothesis-based research typically associated with biomedical research, novel approaches in which the exploratory concept that the tissue and clinical phenotype is quite likely a consequence of a 'genetic portfolio' working in concert, in contrast with the output of a single master gene, are being pursued. Sophisticated computer modeling such as Bayesian algorithms [5] have identified new and biologically plausible genetic profiles that in turn can be utilized to develop new hypotheses followed by prospective investigation.

It is now also an opportune time for new directions in research such that selected aspects of the molecular pain component be integrated into this modeling, given the prominence of oral mucosal pain in the clinical setting [2]. The scientific paradigm is well positioned to integrate molecular (e.g., endothelin-1) [25••] and clinical cancer pain models [26] with oral mucositis pathogenesis and clinical management. Unlike symptoms associated with gastrointestinal mucosal injury, oral pain is the prototypic symptom that can be sufficiently severe as to cause delays and/or dose reductions of subsequent chemotherapy cycles as well as hospitalization with associated, typically expensive, supportive care interventions.

The complexity of this pathobiology thus warrants creation of innovative systems biology technology. A key concept in this regard is that the whole is greater than the sum of the parts, and that a systems biology platform can identify previously unidentified relationships among molecular, cellular, and tissue injury [10]. Analytic platforms utilized in research settings other than mucosal injury in cancer are now be customized and applied to mucositis research. This approach holds high promise for delineating key network hub pathways involved in the pathobiology as well as prioritizing the opportunities for potential perturbation via drugs and/or biologics that could in turn mitigate the toxicity $[2,10]$.

The evolution of this field has dramatically escalated over the past decade, with strong signs of continued, expanded progress in the field. This foundation in research as well as clinical impact is in turn enhancing the ability to address a more recently emergent expression of mucosal injury in oncology patients, namely the unique oral mucosal lesions associated with targeted cancer therapies [1, 15-17, 27-29]. These therapeutics comprise several different classes of molecular activity, including inhibitors of either mammalian target of rapamycin (mTOR) or angiogenesis, epidermal growth factor receptors (EGFR), multikinase Abl pathways, or human epidermal growth factor receptor 2 (HER2).

The resultant oral mucosal toxicity is uniquely different from that of mucositis caused by conventional cancer treatment regimens such as high-dose chemotherapy or head and neck radiation.

\section{Epidemiology}

Oral mucositis incidence and severity is based on a number of variables in chemotherapy patients, including mechanism of action of the drug and intensity of dosing regimen as well as genetic-based mucosal susceptibility (e.g., genetic polymorphisms) [30]. Promoters of this injury are also multi-faceted, and include nutritional status, oral mucosal infection, mechanical trauma, and anti-emetic drug-induced salivary hypofunction.

As cited previously, targeted cancer therapeutics have also been demonstrated to cause a unique phenotype of oral mucosal injury. These lesions emerged as essentially unanticipated side effects, despite the precise and molecularly directed mechanisms of action of the biologics. The oral ulcerations resemble aphthous stomatitis in their appearance, with a characteristic macular pseudomembranous center surrounded by an intensive erythematous halo. In this clinical context they have thus been termed 'mTOR inhibitor-associated stomatitis (mIAS)' [17]. 


\section{Clinical Impact}

Unlike symptoms associated with gastrointestinal mucositis, pain is perhaps the most clinically impactful symptom for oral mucositis. The severity of pain, versus objective injury of erythema and/or ulceration, is associated with significant adverse outcomes such as chemotherapy dose delay or reduction, hospitalization, need for narcotic pain control, and parenteral nutritional support. Interestingly, there is not $100 \%$ concordance across oral mucositis patients relative to their pain reporting in relation to objective tissue injury. Even mild inflammatory oral mucosal changes in some patients can thus result in patient-based reports of moderate-to-severe oral pain that warrant extensive and expensive supportive care interventions.

Cancer patients receiving targeted therapies present an interesting basis for comparison in this regard. Elting et al. has reported that oral mucosal lesions, albeit low grade in severity, most frequently occurred in patients treated with sorafenib, sunitinib, bevacizumab, or erlotinib [1]. In selected settings the lesions can adversely impact cancer therapy. For example, a recent systematic review of 44 studies of mTOR inhibitors demonstrated that the oral lesions caused $27.3 \%$ of dose reductions and $13.1 \%$ of dose discontinuations [31].

\section{Assessment}

There are a variety of assessment scales that can be utilized in the clinical setting to assess oral mucosal injury caused by chemotherapy [9]. These scales can in turn set the stage for treatment. Assessment instruments such as the World Health Organization (WHO) scale for oral mucositis or the National Cancer Institute Common Terminology Criteria for Adverse Events (NCI-CTCAE) [32] are two of the prototypic scales that have been utilized over the past many years in both the clinical research and clinical-care settings.

World Health Organization (WHO) scale for oral mucositis

Grade $0=$ No oral mucositis

Grade $1=$ Erythema and soreness

Grade $2=$ Ulcers, able to eat solids

Grade 3 = Ulcers, requires liquid diet (due to mucositis)

Grade 4 = Ulcers, alimentation not possible (due to mucositis)

National Cancer Institute Common Terminology

Criteria for Adverse Events version 4.03

Grade 1 = Asymptomatic or mild symptoms; intervention not indicated
Grade 2 = Moderate pain; not interfering with oral intake; modified diet indicated

Grade 3 = Severe pain; interfering with oral intake

Grade 4 = Life-threatening consequences; urgent intervention indicated

Grade $5=$ Death

These scales typically combine assessment of type of oral mucosal injury (e.g., erythema, ulceration), oral symptoms (e.g., pain), and degree of functional disturbance due to that pain (e.g., type of dietary intake). Oral mucositis typically initially presents clinically approximately 5-7 days after first dose of chemotherapy, with the patient reporting a sensation of oral mucosal warmth and/or burning. Overt erythema with eventual ulceration classically occurs approximately 10 days after first dose of chemotherapy, with wound resolution occurring in the 2-4 weeks after last dose of chemotherapy. The clinical appearance of the lesions combined with the temporal assessment in relation to chemotherapy dosing is usually sufficient at the clinical level to establish the clinical diagnosis of oral mucositis.

In contrast to this trajectory, oral mucosal lesions caused by the targeted cancer agents may in select cases first develop several or weeks or even a few months after initial dose exposure. Despite this variation in temporal expression versus that of mucositis caused by conventional chemotherapy, clinical diagnosis can usually be achieved by history and clinical examination, as is the case in conventional chemotherapy patients $[6,17]$.

There is, at present, no fully validated assessment scale for these lesions. Given the clinical importance of these lesions in oncology practice, Boers-Doets and Lalla have proposed a new scale in which both a subjective measurement of patient-reported pain and an objective component measuring duration of lesions has been incorporated [33]. This tool, pending further development and validation, may enhance both the research as well as clinical care of patients with these lesions. For example, the design of this scale contributes to the design of clinical decision making such that dose modification will be considered only when Grade 3 severity occurs in both the subjective and objective domains, as listed below.

Subjective

Grade $0=$ No oropharyngeal pain attributed to mIAS

Grade $1=$ Oropharyngeal pain attributed to mIAS, with average oropharyngeal pain score (over the last 24 hours) reported as $\leq 2$ on a $0-10$ scale

Grade 2 = Oropharyngeal pain attributed to mIAS, with average oropharyngeal pain score (over the last 24 hours) reported as $\leq 5$ on a $0-10$ scale 
Grade 3 = Oropharyngeal pain attributed to mIAS, with average oropharyngeal pain score (over the last 24 hours) reported as $\geq 6$ on a $0-10$ scale

\section{Objective}

Grade $0=$ No visible mIAS (i.e., no erythema and no ulceration, attributed to mIAS, in the oropharyngeal area) Grade $1=$ Oral and/or pharyngeal erythema, attributed to mIAS, but no ulceration

Grade 2 = Visible oral and/or pharyngeal ulceration(s), attributed to mIAS, of $<7$ days' duration

Grade 3 = Visible oral and/or pharyngeal ulceration(s), attributed to mIAS, with at least one ulceration persisting for $\geq 7$ days

\section{Management}

The Multinational Association of Supportive Care in Cancer/ International Society of Oral Oncology (MASCC/ISOO) developed the first set of evidence-based guidelines for oral and gastrointestinal mucositis in 2004 [34]. Since then, updates have been published in 2007 [35] and most recently in 2013/2014 [3]. The reader is referred to the MASCC/ISOO website for access to the full portfolio of evidence-based recommendations.

Members of other organizations have utilized this foundation provided by MASCC/ISOO in relation to development of mucositis guidelines. Examples of these organizations include:

- European Society for Medical Oncology (ESMO)

- Oncology Nursing Society (ONS)

- National Comprehensive Cancer Network (NCCN).

An important update has emerged over the past two years relative to the specific intervention with palifermin to prevent oral mucositis in HCT patients. Historically, the labeling of palifermin was limited to patients with hematologic malignancy and undergoing high-dose chemotherapy with or without total body irradiation, followed by HCT rescue. The US FDA has more recently determined that palifermin is approved in the following setting [12]:

"...with hematologic malignancy treated with chemotherapy and/or targeted agents, and/or HSCT with or without TBI (local-regional radiotherapy alone not included), and who are anticipated to develop Grade 3 or Grade 4 oral mucositis."
Having said this, palifermin, first approved by the US FDA in December 2004, has not gained the widespread use in HCT settings that was envisioned by researchers and clinicians whose primary investigative interests include oral mucositis. A number of reasons likely exist for this outcome, including cost of the product as well as the view of some oncologists that oral mucositis, although clinically important, does not necessitate a molecularly targeted intervention to reduce its severity.

In contrast, basic oral care (e.g., oral hygiene, oral nonmedicated rinses) are widely advocated as a means to sooth the oral mucosa, promote clearance of food debris and colonizing microflora, and hydrate the tissue. As reported in a recent systematic review by McGuire et al. [36], the literaturebased evidence does not permit development of guidelines relative to this aspect of wound care, including dental care interventions and/or use of water alone, saline, or sodium bicarbonate oral rinses. There can thus be variation across institutions as to the type of basic wound care protocols that are utilized.

Recent reviews have provided guidance relative to oral care in patients who develop mucosal injury secondary to targeted cancer therapies $[6,16]$. In this case, expert opinions represent the state-of-the-science, since no clinical trials have been reported that show superiority of one intervention over another. The following interventions are described in these two reviews.

- Increase the frequency of the bland, non-alcoholic, sodium-containing mouthwash, if necessary up to each hour.

- If patients find the mouthwash painful, they should be advised to use pain medication beforehand.

- Consider sugarless chewing gum or candy, salivary substitutes, or sialogogues in patients with oral dryness.

- Consider topical high-potency corticosteroids first.

- If no resolution, consider intralesional steroid injection and topical clobetasol gel or ointment.

- If highly symptomatic and with recurrent ulcers or esophageal lesions:

- consider systemic corticosteroids as initial therapy to efficiently bring symptom under control

- provide adequate pain management

- for persistent severe pain use more aggressive pain management.

\section{Barriers in the Field}

Although significant progress has been made in this field over the past 15 years, there remain several key barriers to moving ahead. These barriers can be viewed in both the scientific and clinical domains. 
Scientific domain

The central theme of this domain is that the biologic complexity of mucositis provides opportunity for enhancing new knowledge relative to at least three key areas: (i) understanding the biological basis for the lesion, (ii) predicting risk for development of mucositis across different oncology populations, and (iii) identifying a priori patients who will or will not respond to clinical interventions with drugs or biologics for management of the condition [11]. The role of genetic regulation of these processes is paramount in this modeling, as is the opportunity to integrate the biologic and computational sciences in order to comprehensively study these new frontiers. The next steps could involve:

- further delineation of key network hubs and pathways that are central to the pathobiology as well as wound healing;

- enhanced understanding of the role of oral mucosalbased genetic governance of mucosal injury and repair;

- integration of molecular pain modeling, including neuropeptides and endothelin-1, into the pathobiology framework;

- additional studies of the role of the oral microbiome in either protecting against or increasing oral mucositis incidence and severity;

- new studies delineating molecular mechanism relative to oral mucosal injury caused by targeted cancer therapeutics, comparing and contrasting with the conceptual model for mucositis caused by conventional chemotherapy;

- integration of novel systems biology analytic strategies with the biologic sciences in order to permit 'big science' approaches in the study of mucosal injury in cancer patients.

\section{Clinical domain}

The central theme of this domain is to strategically integrate a new generation of mucositis therapeutics into high-quality clinical guidelines together with point-of-care a priori predictive technology. This approach would in turn enhance the clinician's ability to customize mucositis prevention and management for each at-risk oncology patient. There is a need for:

- additional, robust clinical drug and device development;

- incorporation of patient-based risk profiling into clinical trial designs;

- commitment of industry sponsors to address this unmet medical need;
- novel approaches relative to high-quality, evidence-based clinical guidelines, including:

- new approaches for dissemination via contemporary technology platforms;

- measurement of impact of evidence-based clinical guidelines relative to enhanced clinical outcomes while reducing cost of cancer care.

Advances in the scientific and clinical domains as described above could in turn strategically enhance the pace of regulatory approval for new preventive and treatment approaches for this cancer treatment toxicity.

\section{Summary}

There have been strategic advances over the past decade relative to oral mucositis caused by chemotherapy. These advances include new insights into pathobiology, continued development of drugs, biologics, and devices for management, and clinical care guidelines at the expert opinion or systematic review level that provide opportunity for state-ofthe-science clinical care.

As also described in this report, however, barriers exist in association with these opportunities. These barriers include need for enhanced understanding of pathobiology including pain, regulatory approval of novel therapeutics, and enhanced dissemination and assessment of impact of clinical guidelines in oncology settings.

\section{Compliance with Ethics Guidelines}

Conflict of Interest Dr. Douglas Peterson reports no conflict of interest.

Human and Animal Rights and Informed Consent This article does not contain any studies with human or animal subjects performed by the author.

\section{References}

Papers of particular interest, published recently, have been highlighted as:

-• Of major importance

1. Elting LS, Chang YC, Parelkar P, et al. Risk of oral and gastrointestinal mucosal injury among patients receiving selected targeted agents: a meta-analysis. Support Care Cancer. 2013;21(11):3243-54.

2. Jensen SB, Peterson DE. Oral mucosal injury caused by cancer therapies: current management and new frontiers in research. J Oral Pathol Med. 2014;43(2):81-90. 
3.• Lalla RV, Bowen J, Barasch A, et al. MASCC/ISOO clinical practice guidelines for the management of mucositis secondary to cancer therapy. Cancer. 2014;120(10):1453-61. High quality, evidence-based mucositis guidelines updated from original 2004 guidelines and first revision 2007 guidelines.

4. National Cancer Institute $P D Q{ }^{\mathbb{R}}$. Oral mucositis 2014. Available from: http://www.cancer.gov/cancertopics/pdq/supportivecare/ oralcomplications/HealthProfessional/page5.

5. Sonis S, Antin J, Tedaldi M, et al. SNP-based Bayesian networks can predict oral mucositis risk in autologous stem cell transplant recipients. Oral Dis. 2013;19(7):721-7.

6. Peterson D, Bensadoun R-J, Boers-Doets C, et al. Management of oral and gastrointestinal mucosal injury. ESMO clinical practice guidelines for diagnosis, treatment, and follow-up. Ann Oncol., in press.

7. Mougeot JL, Mougeot FK, Peterson DE, et al. Use of archived biopsy specimens to study gene expression in oral mucosa from chemotherapy-treated cancer patients. Oral Surg Oral Med Oral Pathol Oral Radiol. 2013;115(5):630-7.

8. Logan RM, Stringer AM, Bowen JM, et al. Is the pathobiology of chemotherapy-induced alimentary tract mucositis influenced by the type of mucotoxic drug administered? Cancer Chemother Pharmacol. 2009;63(2):239-51.

9. Lalla RV, Saunders DP, Peterson DE. Chemotherapy or radiationinduced oral mucositis. Dent Clin North Am. 2014;58(2):341-9.

10. Peterson D, Srivastava R, Lalla R. Oral mucosal injury in oncology patients: Perspectives on maturation of a field. Oral Dis. 2013 doi: 10.1111/odi.12167.

11. Peterson DE, Keefe DM, Sonis ST. New frontiers in mucositis. In: Govindan R, editor. 2012 ASCO Educational Book. Alexandria: ASCO; 2012. p. 545-51.

12. National Cancer Institute. FDA approval for palifermin 2013. Available from: http://www.cancer.gov/cancertopics/druginfo/fdapalifermin/print.

13. Sonis ST. A biological approach to mucositis. J Support Oncol. 2004;2(1):21-32. discussion 5-6.

14. Sonis ST. New thoughts on the initiation of mucositis. Oral Dis. 2010;16(7):597-600.

15. Pilotte AP, Hohos MB, Polson KM, et al. Managing stomatitis in patients treated with mammalian target of rapamycin inhibitors. Clin J Oncol Nurs. 2011;15(5):E83-9.

16.• Boers-Doets CB, Raber-Durlacher JE, Treister NS, et al. Mammalian target of rapamycin inhibitor-associated stomatitis. Future Oncol. 2013;9(12):1883-92. Contemporary review of oral mucosal injury caused by targeted cancer therapy.

17. Sonis S, Treister N, Chawla S, et al. Preliminary characterization of oral lesions associated with inhibitors of mammalian target of rapamycin in cancer patients. Cancer. 2010;116(1):210-5.

18. Nonzee NJ, Dandade NA, Patel U, et al. Evaluating the supportive care costs of severe radiochemotherapy-induced mucositis and pharyngitis: results from a Northwestern University Costs of Cancer Program pilot study with head and neck and nonsmall cell lung cancer patients who received care at a county hospital, a Veterans Administration hospital, or a comprehensive cancer care center. Cancer. 2008;113(6):1446-52.
19. Sonis ST, Oster G, Fuchs H, et al. Oral mucositis and the clinical and economic outcomes of hematopoietic stem-cell transplantation. J Clin Oncol. 2001;19(8):2201-5.

20. Sonis ST. Pathobiology of oral mucositis: novel insights and opportunities. J Support Oncol. 2007;5(9 Suppl 4):3-11.

21. Sonis ST. Oral mucositis. Anticancer Drugs. 2011;22(7):607-12.

22. Sonis ST, Elting LS, Keefe D, et al. Perspectives on cancer therapyinduced mucosal injury: pathogenesis, measurement, epidemiology, and consequences for patients. Cancer. 2004;100(9 Suppl): 1995-2025.

23. Al-Dasooqi N, Sonis ST, Bowen JM, et al. Emerging evidence on the pathobiology of mucositis. Support Care Cancer. 2013;21(11): 3233-41.

24. Stringer AM. Interaction between host cells and microbes in chemotherapy-induced mucositis. Nutrients. 2013;5(5):1488 99.

25.• Schmidt BL. The neurobiology of cancer pain. Neuroscientist 2014. doi:10.1177/1073858414525828. State of science review of preclinical and clinical research of cancer pain, with potential translation into oral mucositis pain modeling in the future.

26. Goodwin PJ, Bruera E, Stockler M. Pain in patients with cancer. J Clin Oncol. 2014;32(16):1637-9.

27. Boers-Doets CB, Epstein JB, Raber-Durlacher JE, et al. Oral adverse events associated with tyrosine kinase and mammalian target of rapamycin inhibitors in renal cell carcinoma: a structured literature review. Oncologist. 2012;17(1):135-44.

28. de Oliveira MA, Martins EMF, Wang Q, et al. Clinical presentation and management of mTOR inhibitor-associated stomatitis. Oral Oncol. 2011;47(10):998-1003.

29. Watters AL, Epstein JB, Agulnik M. Oral complications of targeted cancer therapies: a narrative literature review. Oral Oncol. 2011;47(6):441-8.

30. Sonis S, Haddad R, Posner M, et al. Gene expression changes in peripheral blood cells provide insight into the biological mechanisms associated with regimen-related toxicities in patients being treated for head and neck cancers. Oral Oncol. 2007;43(3):289300 .

31. Kwitkowski VE, Prowell TM, Ibrahim A, et al. FDA approval summary: temsirolimus as treatment for advanced renal cell carcinoma. Oncologist. 2010;15(4):428-35.

32. National Cancer Institute Common Terminology for Adverse Events. http://evs.nci.nih.gov/ftp1/CTCAE/About.html. Accessed 5 Jun 2014.

33. Boers-Doets CB, Lalla RV. The mIAS scale: a scale to measure mTOR inhibitor-associated stomatitis. Support Care Cancer. 2013;21(S1):S140.

34. Rubenstein EB, Peterson DE, Schubert $M$, et al. Clinical practice guidelines for the prevention and treatment of cancer therapy-induced oral and gastrointestinal mucositis. Cancer. 2004;100(9 Suppl):2026-46.

35. Keefe DM, Schubert MM, Elting LS, et al. Updated clinical practice guidelines for the prevention and treatment of mucositis. Cancer. 2007;109(5):820-31.

36. McGuire DB, Fulton JS, Park J, et al. Systematic review of basic oral care for the management of oral mucositis in cancer patients. Support Care Cancer. 2013;21(11):3165-77. 